\title{
Katzen in der Kleintierpraxis - Maßnahmen, um den Stress zu reduzieren
}

\author{
Marietta Bartels
}

Die Beliebtheit von Katzen nimmt insbesondere in den Städten ständig zu. Dementsprechend steigt die Anzahl der Katzenpatienten in der Kleintierpraxis. In Bezug auf Verhalten, Krankheiten und Stressempfindlichkeit sind Katzen auf keinen Fall als „kleine Hunde“ zu betrachten. Auch die Katzenbesitzer unterscheiden sich in ihren Erwartungen an den Tierarzt erheblich von den Hundebesitzern: Sie sind feinfühliger, stellen mehr Fragen und sind besser informiert. Damit die Tierarztpraxis und das Praxisteam den speziellen Bedürfnissen der Katzenpatienten und den Ansprüchen der Katzenbesitzer gerecht werden können, sind einige Maßnahmen nötig.

\section{Der Katzenpatient}

Abhängig von ihrer Sozialisierung und ihrer Erfahrung im Umgang mit Menschen reagiert jede Katze unterschiedlich auf neue Eindrücke und Stress. Das natürliche Verhalten der Katze besteht primär darin, einer Gefahr aus dem Weg zu gehen und das nächste sichere Ver- steck aufzusuchen. In sehr seltenen Fällen - bedingt durch schlechte oder schmerzhafte Erlebnisse - reagiert eine Katze mit aggressivem Verhalten.

Bevor die Katze in die Praxis kommt, erlebt sie eine ganze Reihe stressiger Situationen. Zuerst sperrt sie der besorgte Besitzer in einen Transportbehälter ein. Der Mangel an Fluchtmöglichkeiten verursacht Angst und Stress. Der Transport gestaltet sich fast immer als schaukelnd und schüttelnd. Damit wird der Katze quasi der Boden unter den Pfoten weggerissen: ein erneuter Stressauslöser. Im Wartezimmer wird sie mit fremden Gerüchen und Geräuschen konfrontiert. Im schlimmsten Fall bellt der Hund neben ihr. Diese Situation verursacht zusammen mit dem Eingesperrtsein im Transportkorb eine ungeheure Angst.

Zusammenfassend hat die Katze, bevor sie im tierärztlichen Sprechzimmer überhaupt angekommen ist, eine unglaublich belastende Zeit hinter sich gebracht. Zudem ist sie eventuell noch krank.

\section{Das Wartezimmer}

Um den Stress zu reduzieren, sollte das Wartezimmer eine möglichst freundliche und ruhige Atmosphäre ausstrahlen. Optimal wären getrennte Wartebereiche für Hunde und Katzen. Diese sind jedoch leider fast nur in Kliniken realisierbar. Eine räumliche Trennung innerhalb eines Raumes (z. B. durch einen Paravent) bringt keine wesentlichen Vorteile und ist daher nicht empfehlenswert. Wenn keine getrennten Wartebereiche für Hunde und Katzen möglich sind, ist es besonders wichtig, dass Katzen nicht neben Hunden warten müssen. Die im Empfang arbeitenden TFA sollten sich schnell und kompetent bemühen, eine solche Situation zu vermeiden. Sie sollten den Katzenbesitzer baldmöglichst in ein separates Sprechzimmer geleiten. Auch gut organisierte Terminsprechstunden minimieren die Wartezeiten und somit den Stress für die Patienten.

Die immer wiederkehrende Situation eines schnüffelnden, gar bellenden Hundes vor einem am Boden abgestellten Katzenkorb ist der schlimmste Stress, den man einer Katze antun kann. Erhöh-

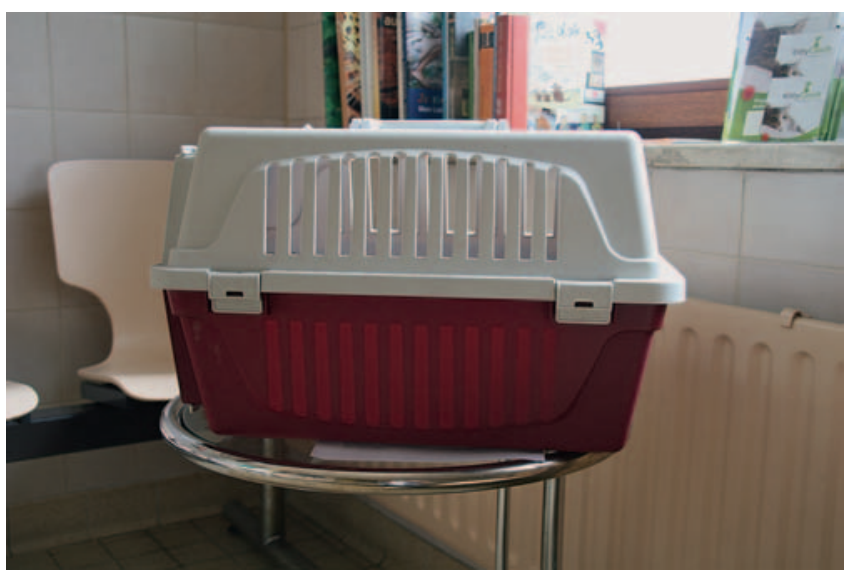

Abb. 1 Erhöhte Abstellmöglichkeiten für Katzenkörbe im Wartezimmer reduzieren den Stress der Patienten. (C) M. Bartels

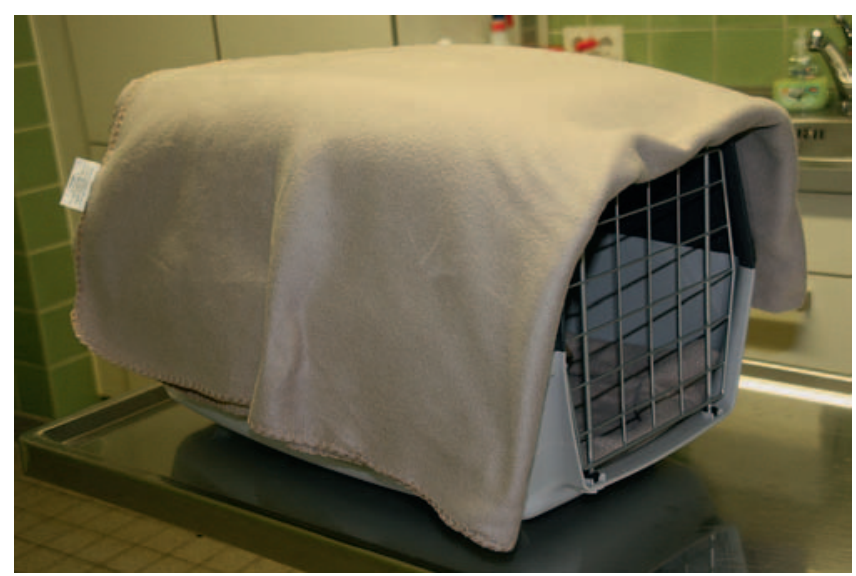

Abb. 2 Eine Decke über dem Transportkorb trägt wesentlich zur Beruhigung der Katze bei. (c) M. Bartels 
te Abstellmöglichkeiten für den Katzenkorb in Form kleiner Tische oder Bretter bieten eine einfache und ideale Lösung für dieses Problem ( $\triangleright$ Abb. 1). Sollten die Katzenbesitzer diese Möglichkeit nicht nutzen und den Katzenkorb auf den Boden stellen, ist es Aufgabe der Mitarbeiter, sie auf eine geeignetere Abstellmöglichkeit hinzuweisen.

Viele Kunden vergessen, eine Decke oder ein Tuch zum Abdecken des Korbes mitzunehmen ( $\triangleright$ Abb. 2). Das Praxisteam sollte daher jedem Katzenbesitzer, dessen Korb nicht zugedeckt ist, sofort eine Decke oder ein Tuch zur Verfügung stellen.

Transportboxen aus Kunststoff, die der Größe der Katze angepasst sind, eignen sich für den Transport zum Tierarzt am besten. Sie sind empfehlenswert, da sie sich vernünftig reinigen lassen. Das direkte Einsprühen der Box mit einem Pheromon-Präparat wird erfahrungsgemäß von manchen Katzen als unangenehm empfunden.

Im Wartezimmer sollten stets ausreichend Zeitschriften, Bücher und Informationsmaterialien speziell für Katzenbesitzer ausliegen.

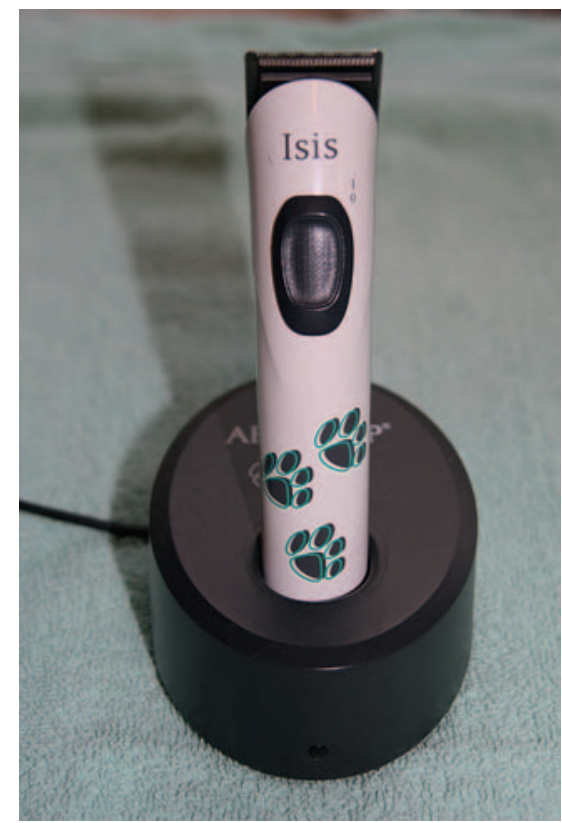

Abb. 3 Eine kleine und sehr leise Schermaschine wird von den Katzen besser toleriert. (c) M. Bartels

\section{Das Sprechzimmer}

Ein Sprechzimmer für Katzen muss ruhig sein und möglichst eine entspannte Atmosphäre vermitteln. Laute Geräusche, z.B. durch Straßenlärm oder klingelnde Telefone, sind zu vermeiden. Die Fenster müssen daher geschlossen bleiben und die Telefone aus diesem Zimmer verbannt werden. Laute, aufgebrachte Stimmen oder lautes Türenöffnen sind ebenfalls ungünstig. Das Praxisteam sollte daran gewohnt sein, sich leise zu verhalten.

Gedämpftes Licht trägt ebenso wie die erwähnte Decke über dem Transportkorb wesentlich zur Beruhigung der Katze bei.

Das Sprechzimmer sollte stets von fremden und für die Katze unangenehmen Gerüchen (Hund, Alkohol, Medikamente, Kot und Urin von anderen Tieren, Schweiß von Menschen) befreit sein.

Idealerweise sollten separate Sprechzimmer für Hunde und Katze benutzt werden. Ist dies nicht realisierbar, muss das Sprechzimmer nach der Untersuchung eines Hundes kurz gelüftet

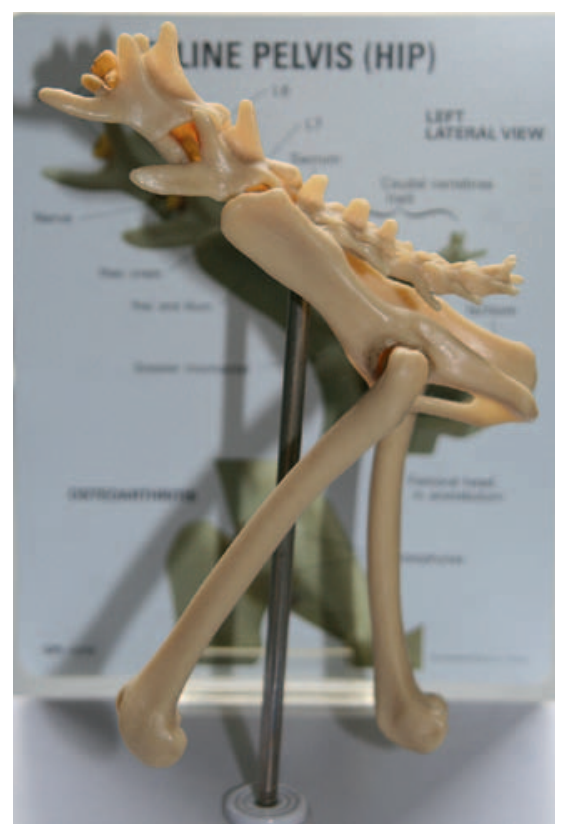

Abb. 4 Felines Anatomiemodell zur Demonstration für Katzenbesitzer. (c) M. Bartels werden, bevor eine Katze untersucht wird.

Der Geruch von Desinfektionsmitteln ist meistens sehr unangenehm für die Katzen. Der Behandlungstisch sollte deshalb nach der Desinfektion sorgfältig trocken gewischt werden. Auch die desinfizierten Hände müssen richtig trocken sein, bevor man mit der Untersuchung beginnt.

Der Einsatz von Pheromon-Präparaten als Zerstäuber im Sprechzimmer trägt zur Entspannung der Patienten bei.

Zur Grundausstattung eines Katzensprechzimmers gehören folgende Elemente:

- Zwei spezielle Waagen, eine für kleine und eine für große Katzen.

- Kleine Wasser- und Fressschalen: Das Wasser ist nach dem Transport - insbesondere an heißen Tagen - unerlässlich und die Leckerlis helfen bei der Untersuchung ängstlicher Patienten. Die Schalen werden samt Inhalt nach jedem Patienten gewechselt und die Näpfe mit kochendem Wasser gereinigt.

- Eine kleine und sehr leise Schermaschine ( $\triangleright$ Abb.3), die von den Katzen besser toleriert wird.

- Ein Körbchen mit Spielzeug, das am besten auf einem der Praxismöbel gelagert wird. Da der Praxisboden letztendlich nie sauber ist, sollten die Katzen nicht auf dem Boden laufen. Sie dürfen sich aber auf dem Behandlungstisch und auf den Praxismöbeln frei bewegen.

- Eine saubere, warme Abdeckung (z.B. ein Handtuch oder eine Decke) für den Behandlungstisch, da Katzen keine Edelstahltische mögen. Die Anwendung von Gummimatten, die nicht nach jeder Untersuchung gewechselt und in der Waschmaschine gereinigt werden, wird aus hygienischen Gründen nicht empfohlen.

- Feline Anatomiemodelle ( $\triangleright$ Abb.4) sowie ein Farbatlas zur Erklärung von Sachverhalten.

Der Röntgen- bzw. Ultraschallraum sollte über spezielle Lagerungshilfen verfügen, die dem Körper und der Größe der Katze angepasst sind. 


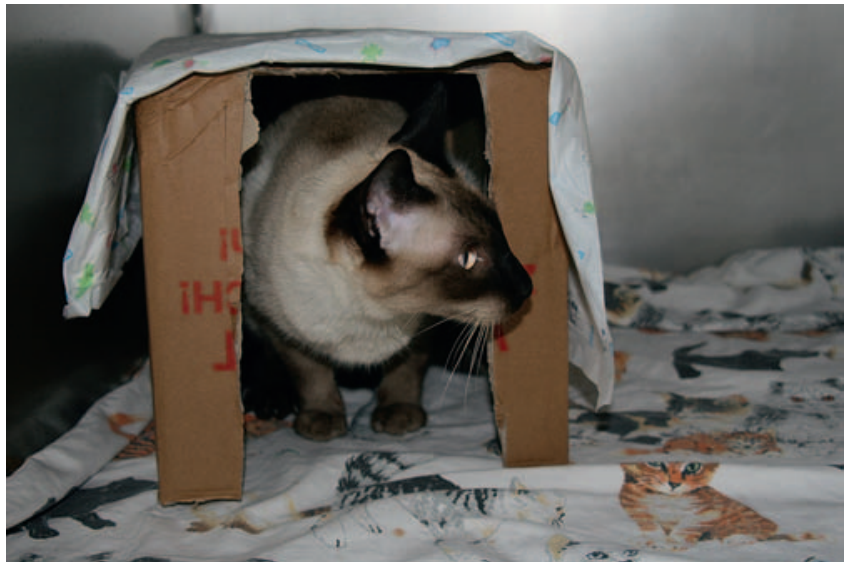

Abb. 5 Im Stationsbereich bietet ein Pappkarton eine ideale Versteckmöglichkeit. @ M. Bartels

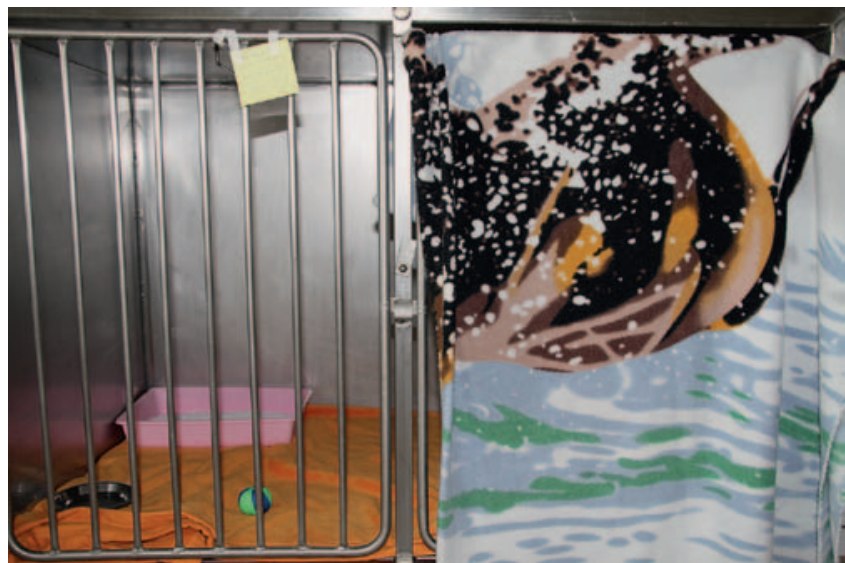

Abb. 6 Eine mit Pheromonen eingesprühte Decke beruhigt besonders ängstliche Katzen. @ M. Bartels

\section{Der Stationsbereich}

Besondere Aufmerksamkeit sollte dem Stationsbereich gewidmet werden.

Getrennte Stationsbereiche für Hunde und Katzen sind unerlässlich: Wenn sie nicht realisierbar sind, sollten zumindest die Operationen von Hunden und Katzen an getrennten Tagen geplant werden.

Jede Katzenbox wird mit weichen, leicht waschbaren Decken oder Tüchern ausgelegt. Bei einem Aufenthalt von mehreren Stunden wird eine kleine Katzentoilette bereitgestellt. Die Besitzer müssen dazu gefragt werden, welche Katzenstreu die Katze gewohnt ist. Zudem sollte ein kleines Kuscheltier oder Kissen zur Verfügung gestellt werden, das anschließend gewaschen wird. Wasser- und Futternäpfe müssen so weit wie möglich von der Katzentoilette entfernt stehen. Falls medizinisch nicht anders indiziert, wird in der Station das gewohnte Futter angeboten. Ein simpler Pappkarton bietet eine praktische Versteckmöglichkeit und wird von vielen Katzen auch gerne als Hochsitz ausprobiert ( Abb. 5).

Der Blickkontakt zwischen einzelnen Katzenboxen ist nicht zwingend zu vermeiden. Manche Katzen unterhalten sich regelrecht auf der Station.

Sollte einer der Patienten im Stationsbereich panisch reagieren, wird eine Decke vor der Box aufgehängt, um diese abzudunkeln. Besonders ängstliche Katzen lassen sich oftmals durch mehrere warme Decken oder Tücher, die vorher mit Pheromonen eingesprüht worden sind, beruhigen ( $\triangleright$ Abb. 6).

Postoperativ sollte eine Wärmequelle in Form eines Wärmekissens nicht fehlen ( $\triangleright$ Abb.7). Um eine Wunde nach einer Operation oder einer Verletzung zu schützen, sollten Katzenbodys anstelle von Halskragen verwendet werden $($ Abb. 8).

Während Hundebesitzer akzeptieren, dass ihr Tier stationär allein bleibt, möchten Katzenbesitzer gerne nach einem chirurgischen Eingriff bei ihrer Katze sitzen ( $\mathbf{A b b} \mathbf{9}$ ). Daher sollten im Stationsbereich hierfür geeignete Sitzmöglichkeiten vorhanden sein.

Für Katzenwelpen sind spezielle Katzenhöhlen durch das Praxisteam vorzu-

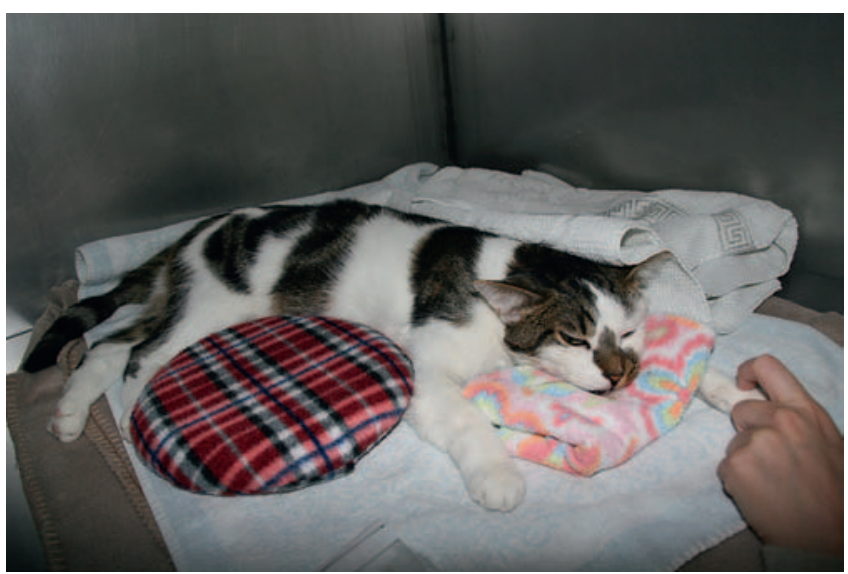

Abb. 7 Wärmekissen als postoperative Wärmequelle. @ M. Bartels

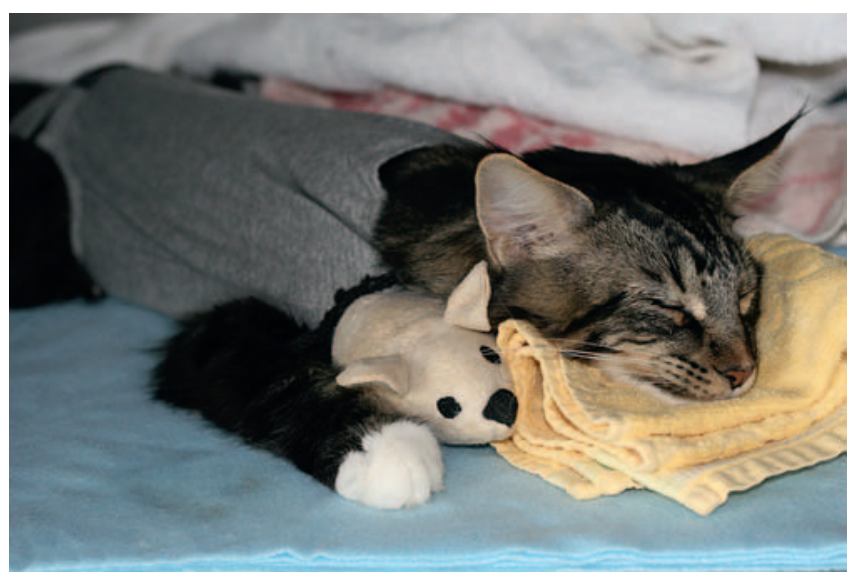

Abb. 8 Ein Katzenbody sollte bei Bedarf anstelle eines Halskragens benutzt werden. (๑) M. Bartels 


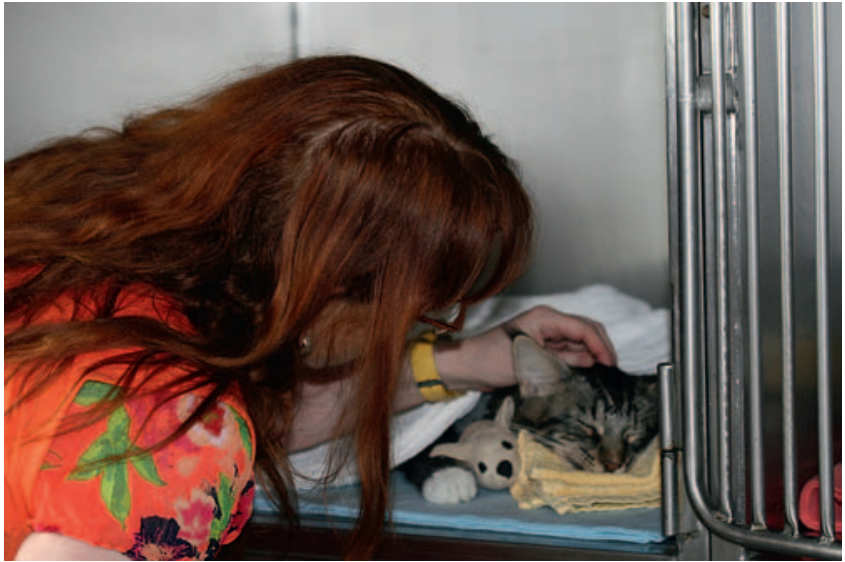

Abb. 9 Katzenbesitzer möchten gerne nach einer Operation bei ihrer Katze sitzen. ( ) M. Bartels

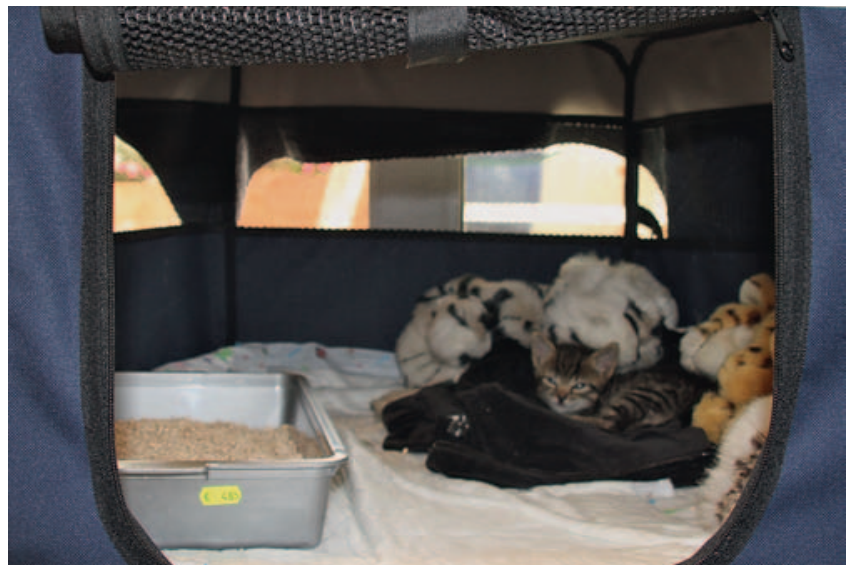

Abb. 10 Spezielle Katzenhöhle für Katzenwelpen. @ M. Bartels bereiten, in denen zusätzlich Decken und Spielzeuge untergebracht werden ( Abb. 10).

\section{Schulung des Praxisteams}

Katzenbesitzer sind meist gut via Internet informiert. Sie haben daher besondere Ansprüche an eine Katzenpraxis. Das Praxisteam sollte für einen liebevollen und ruhigen Umgang mit den Katzenpatienten geschult werden. Katzen mögen es nicht, grob oder hektisch angefasst zu werden. Ein einziger nicht geeigneter Mitarbeiter verdirbt das ganze Team.
Im Idealfall versucht man, die Behandlungstermine auf einige Vormittage/ Nachmittage zu konzentrieren, was allerdings aus Erfahrung nicht immer realisierbar ist.

Die Mitarbeiter am Empfang sollten sich jedoch bemühen, lange Wartezeiten durch die Vergabe sinnvoll geplanter Termine zu vermeiden.

Häufig werden schon vor dem ersten Besuch gezielte Fragen zur Katzengesundheit gestellt. Somit sind die TFA quasi die Eintrittspforte für eine vertrauensvolle Beziehung zur Katze und deren Besitzer. Gezielte Fragen zu Themen wie Verhalten, Fütterung und Ernährung müssen vom ganzen Praxisteam kompetent beantwortet werden. Damit alle Mitarbeiter korrekte und einheitliche Informationen liefern, wird es empfohlen, die wichtigsten Fakten schriftlich zu fixieren und regelmäßige Schulungen für das Praxisteam zu organisieren.

Online zu finden unter

http://dx.doi.org/10.1055/s-0033-1361507

Literatur bei der Verfasserin.

\section{Dr. med. vet. Marietta Bartels}

Spichernstraße 8

40476 Düsseldorf

www.Katzenpraxis-Duesseldorf.de 